\title{
A combination of healthy lifestyle factors is associated with a decreased incidence of chronic kidney disease: a population-based cohort study
}

\begin{abstract}
Minako Wakasugi ${ }^{1}$, Junichiro J Kazama ${ }^{2}$, Suguru Yamamoto ${ }^{3}$, Kazuko Kawamura ${ }^{3}$ and Ichiei Narita $^{3}$
A combination of healthy lifestyle factors is associated with lower risks of coronary heart disease, diabetes and stroke, but little is known about its association with chronic kidney disease (CKD). This study analyzed the effect of a combination of healthy lifestyle factors on the incidence of proteinuria among participants without CKD. Of the 7565 persons aged 40-79 years who participated in the Specific Health Checkups and Guidance System in Sado Island, Japan in 2008, 4902 participants (2015 males) without CKD were included. The healthy lifestyle score was calculated by summing the total number of lifestyle factors for which the participants were at low risk. Low risk was defined as (1) nonsmoker, (2) body mass index (BMI) $<25 \mathrm{~kg} \mathrm{~m}^{-2}$, (3) moderate or less alcohol consumption, (4) regular exercise and (5) better eating patterns. Logistic analysis was used to examine the relationship between the baseline score in 2008 and the development of proteinuria in 2009. Proteinuria developed in $2.2 \%$ of participants (males, 3.2; females, $1.5 \%$ ). Compared with participants with a healthy lifestyle score of 0 to 2, participants with a score of 5 had a lower risk (odds ratio: $0.39,95 \%$ confidence interval: $0.16-0.94$ ), independently of having diabetes, hypertension and hypercholesterolemia. Overall, $47 \%$ of the cases in this cohort could be attributed to lack of adherence to this low-risk pattern. These findings underscore the importance of a healthier lifestyle in preventing CKD. Hypertension Research (2013) 36, 328-333; doi:10.1038/hr.2012.186; published online 22 November 2012
\end{abstract}

Keywords: eating patterns; epidemiology; preventive factors; proteinuria

\section{INTRODUCTION}

A combination of healthy lifestyle factors, including abstaining from smoking, maintaining a BMI of $25 \mathrm{~kg} \mathrm{~m}^{-2}$ or lower, consuming alcohol moderately, exercising regularly and having a healthy diet, produce a significant risk reduction for coronary heart disease, ${ }^{1,2}$ type 2 diabetes mellitus ${ }^{3}$ and stroke. ${ }^{4}$ A clear linear relationship was observed between the risk reduction and the number of healthy lifestyle factors in each study, suggesting that an analysis using a combination of lifestyle factors may capture the influence of multiple health behaviors better than an analysis based on single health behaviors because health behaviors are complex and consist of multiple dimensions.

Chronic kidney disease (CKD) is also closely associated with lifestyle. Several lifestyle factors have been shown to be independently associated with primary prevention of CKD. Obesity, weight gain after maturity and metabolic syndrome increase the risk of CKD, independently of having diabetes mellitus and hypertension..$^{5-7}$ Cigarette smoking is also associated with an increased risk of CKD. ${ }^{5,8-10}$ Alcohol may have both positive and negative effects. While moderate alcohol consumption is associated with a decreased risk, ${ }^{5}$ heavy alcohol consumption is associated with an increased risk of CKD. ${ }^{11}$ However, the combined effects of lifestyle factors on the risk of CKD have not been studied. CKD would also be closely associated with combined lifestyle modification rather than with changes in each factor alone.

Therefore, we hypothesized that a combination of healthy lifestyle factors would be associated with a significant reduction in the risk of CKD. The proportion of CKD that could theoretically be avoided through the simultaneous adoption of multiple types of low-risk behaviors was also estimated.

\section{METHODS}

\section{Study design}

A population-based cohort study of adults aged from 40 to 79 years in Sado City, Japan, who participated in the Specific Health Checkups and Guidance System (SHC) in 2008 and 2009, was performed. Sado City is an island located in the Sea of Japan off the coast of Niigata Prefecture, which is one of the most rapidly aging areas in Japan. The population on the island was 64310 as of

${ }^{1}$ Center for Inter-organ Communication Research, Niigata University Graduate School of Medical and Dental Sciences, Niigata, Japan; ${ }^{2}$ Division of Blood Purification Therapy, Niigata University Medical and Dental Hospital, Niigata, Japan and ${ }^{3}$ Division of Clinical Nephrology and Rheumatology, Niigata University Graduate School of Medical and Dental Science, Niigata, Japan

Correspondence: Dr M Wakasugi, Center for Inter-organ Communication Research, Niigata University Graduate School of Medical and Dental Sciences, Asahimachi 1-757, Chuo-ku, Niigata 951-8510, Japan.

E-mail: minakowa@med.niigata-u.ac.jp

Received 28 February 2012; revised 25 August 2012; accepted 5 September 2012; published online 22 November 2012 
1 October 2008; $>35 \%$ of the population was 65 years or older, of whom half was older than 75 years. This proportion of elderly is almost equal to the projected population of Japan in $2039 . .^{12}$ The details of the SHC have been described elsewhere. ${ }^{6,13}$ In brief, participants answered a self-administered questionnaire that covered their smoking, alcohol consumption, exercise and dietary habits. Then, trained staff measured the height, weight and blood pressure of each participant, after which serum and spot urine samples were collected.

\section{Study participants}

Participants aged from 40 to 79 years without CKD at baseline in 2008 were included. CKD was defined as a GFR $<60 \mathrm{ml} \mathrm{min}^{-1} 1.73 \mathrm{~m}^{-2}$ calculated using the estimated GFR (eGFR) formula for Japanese, ${ }^{14} 1+$ or greater proteinuria on urinalysis, or both. ${ }^{15}$ The data extraction process is shown in the Supplementary information. Of the 7565 participants aged 40 to 79 years screened in 2008, 6429 participants had normal renal function $\left(\mathrm{eGFR} \geqslant 60 \mathrm{mlmin}^{-1} 1.73 \mathrm{~m}^{-2}\right)$ and negative proteinuria on dipstick examination. Of these participants with normal renal function, 4940 participants were re-examined in the 2009 SHC program. A total of 37 participants with missing information on lifestyle factors was excluded, leaving 4902 for this analysis. All of the participants remained anonymous, and the study was conducted according to Japanese privacy protection laws and the ethical guidelines for epidemiological studies published by the Ministry of Education, Science and Culture and the Ministry of Health, Labor and Welfare in 2005 .

\section{Outcomes ascertainment}

The primary end point was incident proteinuria, defined by a dipstick urinalysis score of $1+$ or greater proteinuria (equivalent to $\geqslant 30 \mathrm{mg} \mathrm{dl}^{-1}$ ) because of poor discrimination between negative and trace positive dipstick readings, ${ }^{16}$ at the SHC in 2009 . Because the follow-up duration was relatively short and proteinuria is a significant risk factor for end-stage renal disease, ${ }^{17,18}$ development of proteinuria, but not decreased GFR, was adopted as the end point in the present study.

\section{Lifestyle factors and covariates}

For each lifestyle factor (smoking, BMI, alcohol, exercise and eating patterns), a binary low-risk variable was created, where the participants were given a score of 1 if they met the criteria for low risk and a 0 if otherwise, in accordance with the previous studies. ${ }^{1-4}$ The $a$ priori definition of low risk was based on the current literature and recommended guidelines, as well as on the levels realistically obtainable within the general population. A healthy lifestyle score was calculated by summing the total number of lifestyle factors for which the participants were at low risk. The participants could obtain a healthy lifestyle score from 0 (least healthy) to 5 (most healthy).

For smoking, low risk was defined as not currently smoking. Optimal body weight was defined as a BMI $<25 \mathrm{~kg} \mathrm{~m}^{-2}$, the standard World Health Organization cutoff for healthy weight. For alcohol, average daily alcohol consumption of less than $20 \mathrm{~g}$ was considered low risk, because previous literature showed that average daily alcohol intake of $<20 \mathrm{~g}$ reduced the risk of developing proteinuria in men, ${ }^{5}$ while intake of $>30 \mathrm{~g}$ increased the risk of developing albuminuria. ${ }^{19}$ For exercise, two questions were used. One was 'Are you in the habit of doing exercise to sweat lightly for over $30 \mathrm{~min}$ a time, 2 times weekly, for over a year?' the other was 'In your daily life do you walk or do any equivalent amount of physical activity more than one hour a day?' Low risk was defined as those who answered 'Yes' to both these questions on the basis of a current Japanese guideline. ${ }^{20}$ For eating patterns, two questions were used. One was 'Do you skip breakfast more than 3 times per week?' and the other was 'Do you eat snacks after supper more than 3 times a week?' Low risk was defined as those who answered 'No' to both of these questions.

Diabetes mellitus was defined as the use of insulin or oral antidiabetic medications, hemoglobin Alc $(\mathrm{HbAlc}) \geqslant 6.1 \%$, or both. Hypertension was defined as the use of antihypertensive medications, a systolic blood pressure $\geqslant 140 \mathrm{~mm} \mathrm{Hg}$ and/or a diastolic blood pressure $\geqslant 90 \mathrm{~mm} \mathrm{Hg}$, or both. Hypercholesterolemia was defined as the use of cholesterol-lowering medications, a low-density lipoprotein cholesterol level $\geqslant 140 \mathrm{mg} \mathrm{dl}^{-1}$, or both.

\section{Statistical analysis}

Differences in the distribution of baseline characteristics across the healthy lifestyle score categories were compared using the $\chi^{2}$ test for categorical variables and analysis of variance for continuous variables. Spearman and Pearson correlation coefficients were calculated to evaluate the relationships among the independent variables. The association between each variable of the healthy lifestyle scores and the incidence of proteinuria was first evaluated individually using logistic regression models, and then the association between the healthy lifestyle scores and the incidence of proteinuria was evaluated. A healthy lifestyle score from 0 (least healthy) to 2 was combined to make one category because of the small number of cases. Multivariate-adjusted odds ratios (ORs) and their corresponding 95\% confidence intervals (CIs) were calculated using the category conventionally believed to be the least healthy as the reference group. Two multivariate models were developed. The data were initially adjusted for age and sex. Because hypertension, diabetes and hypercholesterolemia are likely to be intermediate factors in the pathway between lifestyle factors and proteinuria, they were added sequentially to the multivariate models.

To assess the robustness of the main results, several subsidiary analyses were conducted. First, to enhance the association between healthy lifestyle and the outcome, analyses stratified by obesity $\left(\mathrm{BMI} \geqslant 25 \mathrm{~kg} \mathrm{~m}^{-2}\right.$ ) were conducted, because obese participants would modify their lifestyle to lose weight. For participants with obesity, the healthy lifestyle score ranged from 0 to 4 points. Scores from 0 to 2 were combined into one category because of the small number of cases. For participants without obesity, the healthy lifestyle score ranged from 1 to 5 points. Scores from 1 to 3 were combined into one category because of the small number of cases. Second, sensitivity analyses stratified by sex were conducted to establish whether sex had any effect on the relationship, because female sex is associated with a reduced risk of proteinuria. Finally, to avoid the possibility of antiproteinuric effects of medications, subgroup analyses stratified by use or nonuse of medications were conducted.

The population-attributable risk fraction and the corresponding 95\% CIs ${ }^{21}$ were calculated as an estimate of the percentage of developing proteinuria that would not have occurred in this population if all participants had been in the healthiest lifestyle group, on the assumption that there was a causal relation between the risk factors and CKD.

A $P$-value of $<0.05$ was considered significant, and all tests were two-tailed. All statistical analyses were performed with the SPSS for Windows statistical package (Version 18.0; SPSS, Chicago, IL, USA).

\section{RESULTS}

Demographic characteristics of patients

Table 1 shows the associations between various clinical characteristics and healthy lifestyle scores. Participants with a higher healthy lifestyle score had an older age, a lean BMI, a lower blood pressure, lower triglyceride levels, higher high-density lipoprotein cholesterol levels, a lower serum creatinine and a lower eGFR. Males were more likely to have a low healthy lifestyle score. While low-density lipoprotein cholesterol did not differ between the categories, the proportion of cholesterol-lowering medication users was higher in the categories with a high healthy lifestyle score. There were no differences between participants who repeated the program in 2009 and those who did not with regard to baseline characteristics such as sex and BMI, but the latter group was slightly younger and had a high rate of smoking, a higher percentage of medication for diabetes and a higher blood pressure (Supplementary information).

\section{Associations between the healthy lifestyle score and proteinuria} After 1 year of follow-up, 110 (66 males) of the 4902 participants (2015 males) developed proteinuria. When considered individually, there were no apparent associations between each variable of the healthy lifestyle score and the incidence of proteinuria, except BMI (Table 2). There were no significant interactions among these variables. 
Table 1 Clinical characteristics of the 4902 participants by healthy lifestyle score

\begin{tabular}{|c|c|c|c|c|c|c|c|c|}
\hline Characteristics $^{\text {a }}$ & $\begin{array}{c}\text { Total } \\
(\mathrm{N}=4902)\end{array}$ & \multicolumn{6}{|c|}{ Healthy lifestyle score } & $\begin{array}{l}\mathrm{P} \text { for } \\
\text { trend }\end{array}$ \\
\hline Age, years & $66.7(8.4)$ & $64.8(6.1)$ & $58.1(8.9)$ & $62.6(9.5)$ & $65.7(8.7)$ & $67.8(8.0)$ & $68.4(7.0)$ & $<0.0001$ \\
\hline Males, $n(\%)$ & $2015(41.1)$ & $6(100.0)$ & $98(83.1)$ & 345 (69.3) & $636(45.3)$ & $727(32.5)$ & 203 (31.9) & $<0.0001$ \\
\hline Current smoker, $n(\%)$ & $619(12.6)$ & $6(100.0)$ & $97(82.2)$ & $233(46.8)$ & 224 (15.9) & $59(2.6)$ & $0(0.0)$ & $<0.0001$ \\
\hline Body mass index, $\mathrm{kg} \mathrm{m}^{-2}$ & $23.0(3.1)$ & $27.5(1.4)$ & $24.9(3.1)$ & $24.8(3.5)$ & $24.2(3.3)$ & $22.2(2.5)$ & $21.8(2.0)$ & $<0.0001$ \\
\hline \multicolumn{9}{|l|}{ Regular exercise } \\
\hline Exercise to sweat lightly, $n(\%)$ & $1438(29.3)$ & $0(0.0)$ & $6(5.1)$ & $42(8.4)$ & $185(13.2)$ & $569(25.4)$ & $636(100.0)$ & $<0.0001$ \\
\hline Walking $>1 \mathrm{~h}$ per day, $n(\%)$ & $3467(70.7)$ & $2(33.3)$ & $76(64.4)$ & $301(60.4)$ & $935(66.5)$ & $1517(67.8)$ & $636(100.0)$ & $<0.0001$ \\
\hline \multicolumn{9}{|l|}{ Eating pattern } \\
\hline Snacks after supper, $n(\%)$ & $708(14.4)$ & $3(50.0)$ & $40(33.9)$ & $208(41.8)$ & $345(24.6)$ & $112(5.0)$ & $0(0.0)$ & $<0.0001$ \\
\hline Skipping breakfast, $n(\%)$ & $329(6.7)$ & $5(83.3)$ & $59(50.0)$ & 99 (19.9) & $123(8.8)$ & $43(1.9)$ & $0(0.0)$ & $<0.0001$ \\
\hline LDL cholesterol, mg per $100 \mathrm{ml}$ & $128(31)$ & $117(32)$ & $126(31)$ & $125(36)$ & $128(31)$ & $128(31)$ & $129(30)$ & 0.03 \\
\hline Cholesterol-lowering medication, $n(\%)$ & $697(14.2)$ & $0(0.0)$ & $11(9.3)$ & $49(9.8)$ & $198(14.1)$ & $335(15.0)$ & $104(16.4)$ & $<0.0001$ \\
\hline Triglycerides, $\mathrm{mg}$ per $100 \mathrm{ml}$ & $111(78,156)$ & $96(81,261)$ & $134(93,212)$ & $131(90,195)$ & $116(83,165)$ & $105(75,146)$ & $103(74,144)$ & $<0.0001$ \\
\hline $\mathrm{HDL}$ cholesterol, $\mathrm{mg}$ per $100 \mathrm{ml}$ & 57 (14) & $47(10)$ & $58(16)$ & 55 (14) & $56(14)$ & $57(14)$ & $58(14)$ & $<0.0001$ \\
\hline Creatinine, $\mathrm{mg}$ per $100 \mathrm{ml}$ & $0.67(0.13)$ & $0.80(0.11)$ & $0.73(0.12)$ & $0.71(0.13)$ & $0.67(0.13)$ & $0.65(0.12)$ & $0.65(0.12)$ & $<0.0001$ \\
\hline eGFR, $\mathrm{ml} \mathrm{min}-11.73 \mathrm{~m}^{-2}$ & 79 (13) & $78(8)$ & $84(15)$ & $81(13)$ & $79(13)$ & $78(14)$ & 79 (13) & $<0.0001$ \\
\hline \multicolumn{9}{|l|}{ Outcome data } \\
\hline Development of proteinuria, $n(\%)$ & $110(2.2)$ & $0(0.0)$ & $4(3.4)$ & $19(3.8)$ & $41(2.9)$ & $39(1.7)$ & $7(1.1)$ & $<0.0001$ \\
\hline
\end{tabular}

Abbreviations: HDL, high-density lipoprotein; LDL, low-density lipoprotein.

The definitions of the clinical characteristics are described in the text.

a Numbers in the table shown are mean values (s.d.) for continuous variables except triglycerides (median and interquartile range) or numbers (percentages) for categorical variables.

A dose-response relationship was observed between the healthy lifestyle scores and the incidence of proteinuria for all participants $(P<0.0001$ for trend $)$ and for females $(P=0.01$ for trend, Figure 1$)$. A similar trend was observed for males, but it was not significant ( $P=0.08$ for trend). Table 3 shows that participants with a score of 5 (healthiest category) had an age- and sex-adjusted OR of 0.35 (95\% CI: $0.15-0.85)$ for the incidence of proteinuria compared with participants with a score of 0 to 2 . Additional adjustment for the potential consequences of an unhealthy lifestyle (that is, hypertension, diabetes mellitus and hypercholesterolemia) only partially attenuated the risk (OR: 0.39, 95\% CI: 0.16-0.94).

This analysis was repeated with participants who were not obese $\left(\mathrm{BMI}<25 \mathrm{~kg} \mathrm{~m}^{-2}\right.$ ) (Table 4). The association between the healthy lifestyle score and the incidence of proteinuria showed a similar but slightly stronger association compared with the whole study population. Participants with a score of 5 had a multivariate-adjusted OR of 0.40 (95\% CI: 0.17-0.92) for the incidence of proteinuria compared with participants with a score of 1 to 3 . In contrast, the association was not significant among the participants with obesity. Subgroup analysis stratified by sex showed that the point estimates of the OR were similar in both male and female participants compared with the whole study population, although the association was not significant.

Of the participants who currently took antihypertensive medications, the association was similar but stronger than that for the whole study population. The association was not significant among the participants not on antihypertensive medications (data not shown). Of the participants who were not currently taking cholesterollowering medications, the association between the healthy lifestyle score and the incidence of proteinuria was similar to that of the whole study population (data not shown).

\section{Population-attributable risk fraction among the cohorts}

The population-attributable risk fraction was $47 \%$, suggesting that nearly half of the incidence of proteinuria might have been prevented by compliance with the remaining components of the low-healthy lifestyle score in this population. The number of cases was too small, however, to provide separate estimates for the population-attributable risk fraction (95 CI: -8 to $74 \%$ ).

\section{DISCUSSION}

The present study demonstrated that a combination of healthy lifestyle factors was associated with a substantial reduction in the development of proteinuria, even after controlling for well-known biological mediators such as hypertension, diabetes mellitus and hypercholesterolemia. Moreover, this finding was confirmed in subgroup analyses, such as in nonobese individuals or participants on antihypertensive medications. No significant results were obtained in subgroup analyses stratified by sex or obesity. This is probably due to the small number of participants, because the numbers of cases in the reference categories were small, which led to wide CIs. It is also possible that residual confounding by sex might affect the result in the whole study population, because males were more likely to have a low healthy lifestyle score, and female sex was strongly associated with a reduced risk of proteinuria. Although further study is needed to establish the association between the combined healthy lifestyle and $\mathrm{CKD}$, these results also support the possibility that the combined healthy lifestyle could reduce the risk of developing CKD.

The combined effects of lifestyle factors, including abstaining from smoking, maintaining a BMI of $25 \mathrm{~kg} \mathrm{~m}^{-2}$ or lower, moderate or less alcohol consumption, regular exercise and healthy diet, have been reported to produce a significant risk reduction for coronary heart 
Table 2 Multivariate analysis of the relationships between the components of the healthy lifestyle score and the incidence of proteinuria $(N=4902)$

\begin{tabular}{|c|c|c|}
\hline Variable & $\begin{array}{c}\text { Model } 1 \text { odds ratios }{ }^{\text {a }} \\
(95 \% \mathrm{Cl})\end{array}$ & $\begin{array}{c}\text { Model } 2 \text { odds ratios } \\
(95 \% \mathrm{Cl})\end{array}$ \\
\hline \multicolumn{3}{|l|}{ Categories } \\
\hline \multicolumn{3}{|l|}{ Current smoker } \\
\hline Yes (ref) & 1.00 & 1.00 \\
\hline No & $0.67(0.40-1.12)$ & $0.66(0.40-1.10)$ \\
\hline \multicolumn{3}{|l|}{ Body mass index } \\
\hline$\geqslant 25 \mathrm{~kg} \mathrm{~m}^{-2}$ (Ref) & 1.00 & 1.00 \\
\hline$<25 \mathrm{kgm}^{-2}$ & $0.54(0.37-0.81)^{* *}$ & $0.62(0.41-0.93)^{*}$ \\
\hline \multicolumn{3}{|l|}{ Alcohol consumption } \\
\hline$\geqslant 20$ g per day (Ref) & 1.00 & 1.00 \\
\hline$<20$ g per day & $0.89(0.53-1.49)$ & $0.97(0.57-1.65)$ \\
\hline \multicolumn{3}{|l|}{ Regular exercise } \\
\hline No (ref) & 1.00 & 1.00 \\
\hline Yes & $0.90(0.58-1.42)$ & $0.89(0.57-1.40)$ \\
\hline \multicolumn{3}{|l|}{ Eating pattern } \\
\hline Less healthy (ref) & 1.00 & 1.00 \\
\hline Healthy & $0.88(0.55-1.40)$ & $0.87(0.55-1.39)$ \\
\hline \multicolumn{3}{|l|}{ Sex } \\
\hline Male (ref) & 1.00 & 1.00 \\
\hline Female & $0.52(0.34-0.81)^{* *}$ & $0.58(0.37-0.90)^{*}$ \\
\hline \multicolumn{3}{|l|}{ Age, years } \\
\hline 40-49 (Ref) & 1.00 & 1.00 \\
\hline $50-59$ & $0.42(0.17-1.03)$ & $0.37(0.15-0.92)^{*}$ \\
\hline $60-69$ & $0.60(0.29-1.23)$ & $0.50(0.24-1.04)$ \\
\hline 70-79 & $0.74(0.36-1.51)$ & $0.56(0.27-1.17)$ \\
\hline \multicolumn{3}{|l|}{ Hypertension } \\
\hline Yes (ref) & & 1.00 \\
\hline No & & $0.58(0.38-0.87)^{* *}$ \\
\hline \multicolumn{3}{|l|}{ Diabetes mellitus } \\
\hline Yes (ref) & & 1.00 \\
\hline No & & $0.53(0.31-0.90)^{*}$ \\
\hline \multicolumn{3}{|l|}{ Hypercholesterolemia } \\
\hline Yes (ref) & & 1.00 \\
\hline No & & $1.12(0.75-1.69)$ \\
\hline
\end{tabular}

Abbreviation: $\mathrm{Cl}$, confidence interval

${ }^{*} P<0.05,{ }^{* *} P<0.01$.

${ }^{a}$ Adjusted for age, sex and the other components of the healthy lifestyle score.

${ }^{\text {b} A d j u s t e d ~ f o r ~ t h e ~ v a r i a b l e s ~ i n ~ m o d e l ~} 1$ plus hypertension, diabetes and hypercholesterolemia.

The definitions of these factors are described in the text.

disease, ${ }^{1,2}$ type 2 diabetes mellitus ${ }^{3}$ and stroke. $^{4}$ Because CKD is closely associated with these diseases, these results suggest that the combined healthy lifestyle might act in a common pathway to avoid developing these diseases. In addition, a clear linear reduction of risk has been observed based on adherence to the healthy lifestyle factors in each study. In other words, the more these healthy lifestyle factors are adhered to, the greater the reduction of the risks of these diseases. These suggest that not only single health behaviors but also a combination of these behaviors should be achieved to decrease the incidence of these diseases. Of course, an unhealthy lifestyle, such as

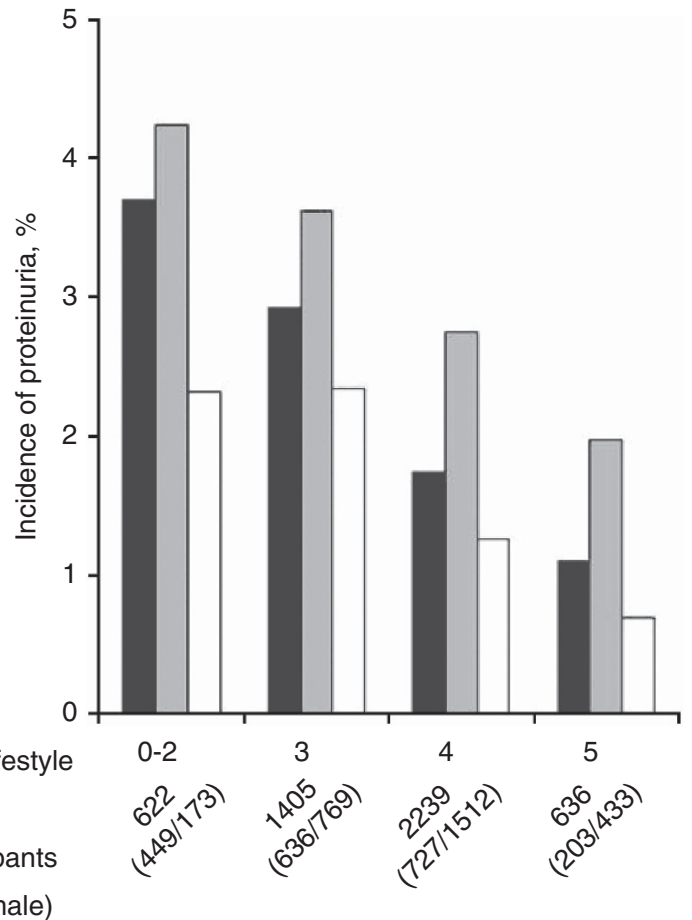

Figure 1 Incidence of proteinuria by healthy lifestyle score. Trends are significant for all participants $(\mathbf{\square} ; P<0.0001)$ and females $(\square ; P=0.01)$, but not significant for males ( $\square ; P=0.08$ ).

poor dietary habits and inadequate physical activity, strongly influences blood pressure, glucose-insulin homeostasis and lipid levels. Furthermore, these lifestyle risk factors also seem to influence novel risk factors such as endothelial dysfunction, oxidative stress, inflammation and other intermediary pathways. ${ }^{22}$ Further research is needed to clarify the combined effect of the healthy lifestyle factors on the primary prevention of these chronic diseases.

With regard to individual components of the healthy lifestyle score, there was no significant reduction of CKD risk except for BMI. Previous literature has shown that obesity, ${ }^{5-7}$ cigarette smoking ${ }^{5,8-10}$ and heavy alcohol consumption ${ }^{11,19}$ are independently associated with an increased risk of CKD. As for smoking and alcohol, the small numbers of cases in the present study might limit the power to detect these possible associations. It is also possible that using binary variables could not detect this association between alcohol and $\mathrm{CKD}$, because alcohol may have both positive and negative effects. $5,11,19$ As for exercise, one study showed that physical inactivity was associated with the risk of CKD in the general population, ${ }^{23}$ but epidemiological studies of this relationship are sparse. As for diet, a classical nutritional factor investigated as a risk factor for $\mathrm{CKD}$ has primarily been salt intake, ${ }^{24}$ but information about eating patterns, such as snacking and skipping breakfast, in the primary prevention of $\mathrm{CKD}$ has been lacking. A growing body of evidence suggests that eating patterns may affect body weight. Although it remains controversial whether snacking contributes to obesity, ${ }^{25}$ a positive association between skipping breakfast and obesity is globally observed, independent of cultural diversity among countries. ${ }^{26-30}$ In addition, skipping breakfast has been found to be associated with increased blood pressure in adolescents $^{31}$ and with health-compromising behaviors in adults and adolescents. ${ }^{32}$ These suggest that these lifestyle factors cooperate with one another, and a combined effect of the healthy lifestyle factors on the primary prevention of $\mathrm{CKD}$ would be 
Table 3 Multivariate analysis of the relationship between the healthy lifestyle score and the incidence of proteinuria $(N=4902)$

\begin{tabular}{|c|c|c|}
\hline Variable & $\begin{array}{l}\text { Age- and sex-adjusted odds ratio } \\
\qquad(95 \% \mathrm{Cl})\end{array}$ & $\begin{array}{l}\text { Multivariate odds ratio } \\
\qquad(95 \% \mathrm{Cl})\end{array}$ \\
\hline \multicolumn{3}{|c|}{ Healthy lifestyle score } \\
\hline 0-2 (Ref) & 1.00 & 1.00 \\
\hline 3 & $0.89(0.52-1.52)$ & $0.94(0.55-1.61)$ \\
\hline 4 & $0.56(0.32-0.98)^{*}$ & $0.63(0.36-1.10)$ \\
\hline 5 & $0.35(0.15-0.85)^{*}$ & $0.39(0.16-0.94)^{*}$ \\
\hline \multicolumn{3}{|l|}{ Sex } \\
\hline Male (ref) & 1.00 & 1.00 \\
\hline Female & $0.52(0.35-0.78)^{* *}$ & $0.58(0.38-0.87)^{* *}$ \\
\hline \multicolumn{3}{|l|}{ Age, years } \\
\hline $40-49$ (ref) & 1.00 & 1.00 \\
\hline $50-59$ & $0.41(0.17-1.00)$ & $0.36(0.15-0.89)^{*}$ \\
\hline $60-69$ & $0.59(0.29-1.21)$ & $0.49(0.24-1.00)$ \\
\hline $70-79$ & $0.72(0.35-1.46)$ & $0.54(0.26-1.12)$ \\
\hline \multicolumn{3}{|l|}{ Hypertension } \\
\hline Yes (ref) & & 1.00 \\
\hline No & & $0.57(0.38-0.85)^{* *}$ \\
\hline \multicolumn{3}{|c|}{ Diabetes mellitus } \\
\hline Yes (ref) & & 1.00 \\
\hline No & & $0.52(0.30-0.87)^{*}$ \\
\hline \multicolumn{3}{|c|}{ Hypercholesterolemia } \\
\hline Yes (ref) & & 1.00 \\
\hline No & & $1.09(0.73-1.63)$ \\
\hline
\end{tabular}

Abbreviation: $\mathrm{Cl}$, confidence interval.

${ }^{*} P<0.05,{ }^{* *} P<0.01$.

aAdjusted for age (years), sex, hypertension, diabetes and hypercholesterolemia. The definitions of these factors are described in the text.

significant, whereas the individual effects of each factor might be weak or insignificant.

The present study also demonstrated that the population-attributable risk fraction was $47 \%$, suggesting that nearly half of the cases of de novo proteinuria might be prevented by comprehensive lifestyle modification. In addition, the present study showed that the participants who were at low risk for the five lifestyle factors had a significant risk reduction, nearly the same as those without hypertension or diabetes. Much effort has been focused on the pharmacologic management of hypertension, diabetes mellitus and hypercholesterolemia for preventing CKD. Although these treatments have been proven to have benefits, they are costly and may have adverse effects. In general, modification of lifestyle is not costly and may not be associated with adverse effects. Therefore, a healthy lifestyle could play a central part in the global war against CKD.

This study has several strengths. To the best of our knowledge, this is the first report about the combined effects of health-related behaviors on the risk of CKD. Because all the participants lived on one small island, the relatively homogenous nature of the cohort would reduce confounding. However, the generalizability to other populations may be limited. Further investigations in other populations are necessary to confirm the present findings. In addition, the population-attributable risk fraction, which provided potentially valuable information regarding the community-level effect of healthy lifestyle factors, was also calculated.
Table 4 Subgroup analyses of the relationship between the healthy lifestyle score and the incidence of proteinuria

\begin{tabular}{|c|c|c|}
\hline $\begin{array}{l}\text { Healthy lifestyle } \\
\text { score }\end{array}$ & $\begin{array}{l}\text { Age- and sex-adjusted } \\
\text { odds ratio }(95 \% \mathrm{Cl})\end{array}$ & $\begin{array}{c}\text { Multivariate odds ratio }{ }^{a} \\
\qquad(95 \% \mathrm{Cl})\end{array}$ \\
\hline \multicolumn{3}{|c|}{ Nonobese individuals $(N=3724)$} \\
\hline $1-3$ (Ref) & 1.00 & 1.00 \\
\hline 4 & $0.55(0.32-0.93)^{*}$ & $0.56(0.33-0.95)^{*}$ \\
\hline 5 & $0.40(0.17-0.94)^{*}$ & $0.40(0.17-0.92)^{*}$ \\
\hline \multicolumn{3}{|c|}{ Obese individuals $(N=1178)$} \\
\hline 0-2 (Ref) & 1.00 & 1.00 \\
\hline 3 & $0.92(0.43-1.97)$ & $0.97(0.45-2.06)$ \\
\hline 4 & $1.60(0.64-3.98)$ & $1.71(0.69-4.27)$ \\
\hline \multicolumn{3}{|l|}{ Males $(N=2015)$} \\
\hline 0-2 (Ref) & 1.00 & 1.00 \\
\hline 3 & $0.87(0.46-1.63)$ & $0.88(0.47-1.66)$ \\
\hline 4 & $0.67(0.34-1.29)$ & $0.71(0.36-1.38)$ \\
\hline 5 & $0.49(0.16-1.42)$ & $0.47(0.15-1.43)$ \\
\hline \multicolumn{3}{|c|}{ Females $(N=2887)$} \\
\hline 0-2 (Ref) & 1.00 & 1.00 \\
\hline 3 & $0.92(0.30-2.775)$ & $1.07(0.35-3.25)$ \\
\hline 4 & $0.46(0.15-1.40)$ & $0.55(0.18-1.69)$ \\
\hline 5 & $0.25(0.05-1.14)$ & $0.31(0.07-1.41)$ \\
\hline \multicolumn{3}{|c|}{ Participants on antihypertensive medications $(N=1505)$} \\
\hline $0-2$ (Ref) & 1.00 & 1.00 \\
\hline 3 & $0.82(0.39-1.73)$ & $0.81(0.38-1.71)$ \\
\hline 4 & $0.38(0.17-0.90)^{*}$ & $0.32(0.17-0.90)^{*}$ \\
\hline 5 & $0.19(0.04-0.87)^{*}$ & $0.13(0.04-0.88)^{*}$ \\
\hline
\end{tabular}

Abbreviation: $\mathrm{Cl}$, confidence interval.

${ }^{*} P<0.05$.

${ }^{a}$ Adjusted for age (years), sex, hypertension, diabetes and hypercholesterolemia. The definitions of these factors are described in the text.

\section{Study limitation}

The present study had several limitations. First, the lifestyle factors other than BMI were determined based on information obtained through self-reporting and may not have been accurate. In addition, the duration of maintaining a healthy lifestyle and the nutritive content of the diet were not evaluated because of a lack of information. Second, despite adjustments for potential confounding factors, residual confounding remains plausible. For example, socioeconomic status was not adjusted for because there was no information. Third, CKD was defined from a single creatinine value and a single measurement of urinary protein because of the nature of an annual health check program. Therefore, it is not possible in this study to confirm whether participants fulfilled CKD criteria for at least a 3-month period. However, the incidence of proteinuria was similar to that in the previous study. ${ }^{8}$ Fourth, the relationship between the healthy lifestyle score and the incidence of proteinuria is influenced by the weight given to each component of the score. We chose to give equal weight to each healthy lifestyle factor, in accordance with previous studies, ${ }^{1-4}$ and this would make the study a clear-cut message for the prevention of coronary heart disease, type 2 diabetes mellitus, stroke and CKD; it provides a simple strategy to minimize the overall incidence of these chronic diseases. However, because the use of equal weights is an imperfect approximation of the underlying biological relationships between lifestyle and CKD, future analyses should examine this issue. Fifth, the present study contained 
small numbers of outcome events and participants, which may explain the wide CIs. Sixth, there may have been selection bias in the study population, because the subjects included in the study received annual physical checks for the second consecutive year; subjects with severe disorders or signs or symptoms tended to attend clinics and hospitals, and thus they did not receive annual physical checks. Finally, the follow-up duration was relatively short. However, as the Specific Health Checkups and Guidance System are ongoing all over Japan, the reproducibility of this study could be assessed by studies with larger sample sizes and longer observation periods if many local governments would work cooperatively. Further research is also needed to assess the relationship between improving the healthy lifestyle factors and the incidence of proteinuria.

In conclusion, a healthy lifestyle was associated with a significant reduction in the risk of $\mathrm{CKD}$, independent of having diabetes mellitus, hypertension and hypercholesterolemia. The present data indicate the possibility that a substantial number of cases of CKD could be prevented by the adoption of a healthier lifestyle. Further investigations in other populations and with longer observation periods are necessary to confirm the present findings.

\section{CONFLICT OF INTEREST}

The authors declare no conflict of interest.

\section{ACKNOWLEDGEMENTS}

We thank the citizens and Sado City Hall for permission to use their data. This study was supported in part by a Grant-in-Aid for Project in Sado for Total Health (PROST) from the Ministry of Education, Culture, Sports, Science and Technology of Japan.

1 Stampfer MJ, Hu FB, Manson JE, Rimm EB, Willett WC. Primary prevention of coronary heart disease in women through diet and lifestyle. N Engl J Med 2000; 343: 16-22.

2 Chiuve SE, McCullough ML, Sacks FM, Rimm EB. Healthy lifestyle factors in the primary prevention of coronary heart disease among men: benefits among users and nonusers of lipid-lowering and antihypertensive medications. Circulation 2006; 114 : 160-167.

3 Hu FB, Manson JE, Stampfer MJ, Colditz G, Liu S, Solomon CG, Willett WC. Diet lifestyle, and the risk of type 2 diabetes mellitus in women. N Engl J Med 2001; 345: 790-797.

4 Kurth T, Moore SC, Gaziano JM, Kase CS, Stampfer MJ, Berger K, Buring JE. Healthy lifestyle and the risk of stroke in women. Arch Intern Med 2006; 166: 1403-1409.

5 Yamagata K, Ishida K, Sairenchi T, Takahashi H, Ohba S, Shiigai T, Narita M, Koyama A. Risk factors for chronic kidney disease in a community-based population: a 10-year follow-up study. Kidney Int 2007; 71: 159-166.

6 Wakasugi M, Narita I, Iseki K, Moriyama T, Yamagata K, Tsuruya K, Yoshida H, Fujimoto S, Asahi K, Kurahashi I, Ohashi Y, Watanabe T. Weight gain after 20 years of age is associated with prevalence of chronic kidney disease. Clin Exp Nephrol 2011; 16: 259-268.

7 Kurella M, Lo JC, Chertow GM. Metabolic syndrome and the risk for chronic kidney disease among nondiabetic adults. J Am Soc Nephrol 2005; 16: 2134-2140.

8 Tozawa M, Iseki K, Iseki C, Oshiro S, Ikemiya Y, Takishita S. Influence of smoking and obesity on the development of proteinuria. Kidney Int 2002; 62: 956-962.
9 Jones-Burton C, Seliger SL, Scherer RW, Mishra SI, Vessal G, Brown J, Weir MR, Fink JC. Cigarette smoking and incident chronic kidney disease: a systematic review. Am J Nephrol 2007; 27: 342-351.

10 Nagasawa Y, Yamamoto R, Rakugi H, Isaka Y. Cigarette smoking and chronic kidney diseases. Hypertens Res 2012; 35: 261-265

11 Shankar A, Klein R, Klein BE. The association among smoking, heavy drinking, and chronic kidney disease. Am J Epidemiol 2006; 164: 263-271.

12 National Institute of Population and Social Security Researchhttp://www.ipss.go.jp/ppnewest/j/newest03/h3_3.html. Accessed 13 September 2011.

13 Kohro T, Furui Y, Mitsutake N, Fujii R, Morita H, Oku S, Ohe K, Nagai R. The Japanese national health screening and intervention program aimed at preventing worsening of the metabolic syndrome. Int Heart J 2008; 49: 193-203.

14 Matsuo S, Imai E, Horio M, Yasuda Y, Tomita K, Nitta K, Yamagata K, Tomino Y, Yokoyama $\mathrm{H}$, Hishida A. Collaborators developing the Japanese equation for estimated GFR. Revised equations for estimated GFR from serum creatinine in Japan. Am J Kidney Dis 2009; 53: 982-992.

15 National Kidney Foundation. K/DOQI clinical practice guidelines for chronic kidney disease: evaluation, classification, and stratification. Am J Kidney Dis 2002; 39(Suppl 1): S1-S266.

16 Harrison NA, Rainford DJ, White GA, Cullen SA, Strike PW. Proteinuria: what value is the dipstick? Br J Urol 1989; 63: 202-208.

17 Iseki K, Iseki C, Ikeyama Y, Fukiyama K. Risk of developing endstage renal disease in a cohort of mass screening. Kidney Int 1996; 49: 800-805.

18 Halbesma N, Kuiken DS, Brantsma AH, Bakker SJ, Wetzels JF, De Zeeuw D, De Jong $\mathrm{PE}$, Gansevoort RT. Macroalbuminuria is a better risk marker than low estimated GFR to identify individuals at risk for accelerated GFR loss in population screening. J Am Soc Nephrol 2006; 17: 2582-2590.

19 White SL, Polkinghorne KR, Cass A, Shaw JE, Atkins RC, Chadban SJ. Alcohol consumption and 5-year onset of chronic kidney disease: the AusDiab study. Nephrol Dial Transplant 2009; 24: 2464-2472.

20 The Office for Lifestyle-Related Diseases Control, General Affairs Division, Health Service Bureau, Ministry of Health, Labour and Welfare of Japan. Exercise and Physical Activity Guide for Health Promotion 2006- To Prevent Lifestyle-related Diseases<Exercise Guide 2006> Prepared in August, 2006 http://www.nih.go.jp/eiken/ programs/pdf/exercise_guide.pdf. Accessed 13 September 2011.

21 Rothman KJ, Greenland S and Lash TL (eds). Modern Epidemiology. 3rd edn. pp 295-297 (Lippincott-Raven, Philadelphia, 2008).

22 Moinuddin I, Leehey DJ. A comparison of aerobic exercise and resistance training in patients with and without chronic kidney disease. Adv Chronic Kidney Dis 2008; 15: 83-96.

23 Stengel B, Tarver-Carr ME, Powe NR, Eberhardt MS, Brancati FL. Lifestyle factors, obesity and the risk of chronic kidney disease. Epidemiology 2003; 14: 479-487.

24 de Francisco AL, Fresnedo GF, Palomar R, Piñera C, Arias M. The renal benefits of a healthy lifestyle. Kidney Int 2005; 68(Suppl 99): S2-S6.

25 Chapelot $\mathrm{D}$. The role of snacking in energy balance: a biobehavioral approach. J Nutr 2011; 141: 158-162.

26 Ma Y, Bertone ER, Stanek 3rd EJ, Reed GW, Hebert JR, Cohen NL, Merriam PA, Ockene IS. Association between eating patterns and obesity in a free-living US adult population. Am J Epidemiol 2003; 158: 85-92.

27 Marín-Guerrero AC, Gutiérrez-Fisac JL, Guallar-Castillón P, Banegas JR, RodríguezArtalejo F. Eating behaviours and obesity in the adult population of Spain. Br J Nutr 2008; 100: 1142-1148.

28 Berg C, Lappas G, Wolk A, Strandhagen E, Torén K, Rosengren A, Thelle D, Lissner L. Eating patterns and portion size associated with obesity in a Swedish population. Appetite 2009; 52: 21-26.

29 Huang CJ, Hu HT, Fan YC, Liao YM, Tsai PS. Associations of breakfast skipping with obesity and health-related quality of life: evidence from a national survey in Taiwan. Int J Obes (Lond) 2010; 34: 720-725.

30 Horikawa C, Kodama S, Yachi Y, Heianza Y, Hirasawa R, lbe Y, Saito K, Shimano H, Yamada N, Sone H. Skipping breakfast and prevalence of overweight and obesity in Asian and Pacific regions: a meta-analysis. Prev Med 2011; 53: 260-267.

31 Kollias A, Antonodimitrakis P, Grammatikos E, Chatziantonakis N, Grammatikos EE, Stergiou GS. Trends in high blood pressure prevalence in Greek adolescents. J Hum Hypertens 2009; 23: 385-390.

32 Keski-Rahkonen A, Kaprio J, Rissanen A, Virkkunen M, Rose RJ. Breakfast skipping and health-compromising behaviors in adolescents and adults. Eur J Clin Nutr 2003; 57: 842-853.

Supplementary Information accompanies the paper on Hypertension Research website (http://www.nature.com/hr) 